\title{
Track for surveys
}

(c) Springer-Verlag GmbH, DE 2021

The cybersecurity field has grown significantly in the past three decades and is still growing rapidly, as evidenced by the number of journals, conferences, and publications in the field and the rate of growth of these numbers. As a result, the extent of the literature on information and cyber security calls for putting results in context, so that researchers and other professionals may stay abreast of developments; such context can be efficiently provided by survey and overview papers. Therefore, it is not surprising that the number of published surveys in the field grows steadily in the last 5 years. Still, no venue dedicated to publishing exclusively such research works exists.

In order to fill this gap, the International Journal of Information Security has decided to establish a new track for surveys. The track focuses on integrating and adding understanding to the existing literature on all aspects of information and cyber security, by publishing survey papers that summarize and analyze the existing literature on a topic. IJIS aims to become the premier source of peer-reviewed, comprehensive surveys, and pointers to further sources.

For further information, visit the journal homepage: https://www.springer.com/journal/10207.

Publisher's Note Springer Nature remains neutral with regard to jurisdictional claims in published maps and institutional affiliations. 